\title{
EXPERIMENTAL ANALYSIS OF WEAR OF DENTAL MILLS
}

\author{
Alexandru Saracin ${ }^{1}$, Petru Cardei $^{2}$, Ion Saracin ${ }^{3}$, Gheorghe Voicu ${ }^{1}$, Ilie Filip ${ }^{1}$ \\ ${ }^{1}$ University Politehnica of Bucharest, Romania; ${ }^{2}$ National Institute of Research-Development for \\ Machines and Installations Designed to Agriculture and Food Industry, Romania; ${ }^{3}$ University of \\ Craiova, Romania \\ saracin.alex@gmail.com,petru_cardei@yahoo.com,ion_saracin@yahoo.com, \\ ghvoicu_2005@yahoo.com, ilie.filip@yahoo.com
}

\begin{abstract}
The paper presents experimental results regarding the main parameters that give information on the wear of the dental mills. The study of the phenomenon of wear of dental mills by experimental methods aims to highlight a multitude of parameters that influence the interaction between the dental mills and the material to be milled. It presents such a set of parameters that define the process and, based on the results, an eventual hierarchy of the influence of these parameters on the milling process is analyzed. The phenomenon of milling wear has been studied in various working regimes generated by the variation of the milling rotation speed and pressing force, measuring at equal time intervals of one hour the main parameters that can provide quantitative and qualitative information about the milling wear: the angle of sharpening, the angle of placement, the angle of clearance, the area of the circle at the tip of the tooth, the radius of the circle at the tip of the tooth in crosssection, the radius of curvature of the release channel, the mass of material lost by the body of the mill. Also, this paper briefly presents the general framework in which the experiments are performed, the milling cutters and the milled material, the working regime. After selecting the set of parameters in which the phenomenon is to be described, the variation of these parameters in relation to time is analyzed. Discussions and conclusions use graphical representations of the experimental data to configure statistical analysis, to develop a complete experimental plan to record any parameters that were not monitored in these early experiments, and also to suggest future statistical or deterministic models of the phenomenon. The deterministic mathematical modelling, towards which the material in this paper tries to orient the future researches, starts from the observation of a possible asymptotic damping of the aggressiveness of the dental mills towards the material and from the expression in terms of the theory of control systems. The mathematical models that are obtained starting from the structured experimental data and commented in this article will be used to investigate the possibilities of improving or optimizing the working regimes of the dental cutters. Both statistical and deterministic models will be used.
\end{abstract}

Keywords: dental, mill, wearing, analysis, experimental.

\section{Introduction}

Rapid wear and tear of dental mills leads to higher prices for laboratory work and dental operations. Being tools created by the fine mechanics, by means of very fine operations and with expensive materials, their rapid wear has led to some studies in the hope that a prolongation of their life or even an optimization of their functioning can be obtained.

The problem of wear of dental mills is a problem analyzed in the last decades, between the reference works being: [1-3]. It also performs a series of works in related fields, regarding instruments of the same type (milling drills, drilling mills, etc.), for example [4-7]. We have approached the problem of dental mills for practical final purposes (optimization or improvement of the operating regimes, of the constructive solutions, possibly of the materials from which they are built, the formulation of criteria for removing the used mills). The phenomenon of dental milling is a classic phenomenon in physics, common in metal cutting theory, in parts wear theory, in drilling processes, in the intimate vicinity of the friction theory. Therefore, some laws of the phenomenon of wear that occurs at the contact of bodies can be used as a source of inspiration. However, for the purposes of application, a list of important parameters must be designated in describing the phenomenon and with which effective combinations can be created to estimate the efficiency of the working process of the dental mills.

\section{Materials and methods}

The material with which the experiments were performed consists of the dental mills tested in these experiments. Some types of mills tested in the experiments are presented in Table 1, together 
with some of their characteristics. The experimental stand for the testing of dental mills, together with its component parts or annexes, appears in the [8].

The method of experimentation, of testing working of the milling cutters, was provided before the actual tests, a series of mechanical and chemical analysis of the materials used. Because milling is a process that depends not only on the material of the milling machine, but to the same extent on the milled material, the mechanical and chemical characterization of the used dental materials was also necessary.

Table 1

Types of dental mills tested and their main characteristics

\begin{tabular}{|c|c|c|c|c|c|c|c|c|c|}
\hline Mill type & F1.1 & F1.2 & F1.3 & F2.1 & $\mathrm{F} 2.2$ & F2.3 & F3.1 & F3.2 & F3.3 \\
\hline Image & & & & & & & & & \\
\hline Mass, & 187 & 183 & 485 & 251 & 51 & 252 & 122 & 121 & 120 \\
\hline Total length, $\mathrm{mm}$ & 52.4 & 52.8 & 52.9 & 45.2 & 45,1 & 44.8 & 44.3 & 44.2 & 44.3 \\
\hline $\begin{array}{l}\text { Length of the active } \\
\text { part, } \mathrm{mm}\end{array}$ & 14.4 & 14.5 & 14.6 & 4.4 & 4.3 & 4.4 & - & - & - \\
\hline Average diameter, $\mathbf{m m}$ & 5.4 & 5.3 & 5.4 & 2.0 & 1.7 & 1.9 & - & - & - \\
\hline $\begin{array}{l}\text { Maximum diameter, } \\
\text { mm }\end{array}$ & 6.2 & 6.0 & 6.3 & 2.2 & 1.9 & 2.1 & 1.1 & 1.1 & 1.1 \\
\hline
\end{tabular}

The chemical and structural analyzes of the ends of the dental mills are described, together with the results, in [8]. Also in [8] there are data and images of the instruments with which they worked, as well as of the methods used to perform the mentioned analyzes. The results of the hardness tests are presented in the same paper [8].

\section{Results and discussion}

During the test program, four-hour milling tests were performed for three rotational speeds of the milling head, measurements being made after each hour of operation. Without exception, after four hours of operation, it was found that for any pressing intensity, the milling machine no longer advances in the material. The list of the process parameters followed in the experimental tests is given in Table 2. In the same table the units of measurement used for these parameters are given.

The results of the experimental tests consist of the numerical records of the main input, command and output parameters, listed in Table 2. Initially, they were recorded in numerical form, but for ease of interpretation we graphically represented the dependence of some of the parameters in relation to others. The graphical representations are given in Fig. 1-7.

Table 2

Process parameters, name, notations and units of measure

\begin{tabular}{|l|c|c|}
\hline \multicolumn{1}{|c|}{ Parameter } & Notation & Units \\
\hline Time & $T$ & seconds, minutes, hours \\
\hline Mass lost through milling wear & $m_{f w}$ & degrees \\
\hline Settlement angle & $\alpha$ & degrees \\
\hline Clearance angle & $\gamma$ & degrees \\
\hline Sharpening angle & $\beta$ & $\mu \mathrm{m}$ \\
\hline Advance & $a$ & $\mathrm{~m}^{2}$ \\
\hline Area of the circle at the top & $A_{c p}$ & $\mathrm{~m}$ \\
\hline Radius of the circle at the top & $R_{c p}$ & $\mathrm{rpm}$ \\
\hline Rotation speed of the milling head & $n$ & \\
\hline
\end{tabular}




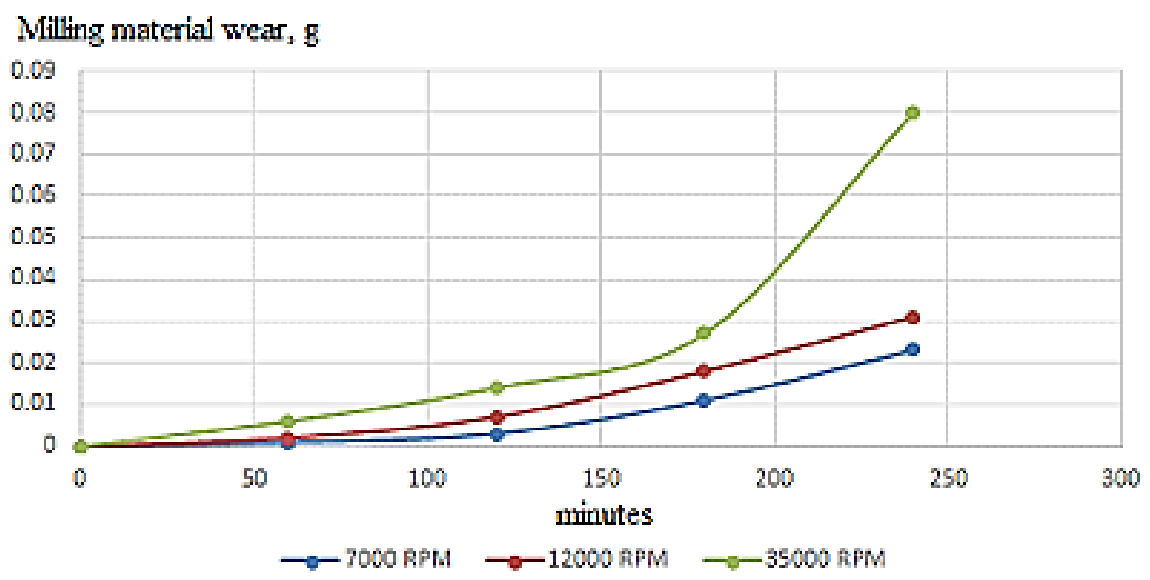

Fig. 1.Time dependence of the wear mass from the dental mill for three values of the milling speed

Fig. 1 shows the variation of the material lost by the dental cutter due to wear as a function of time for three rotational speeds of the dental mill head. It is observed that the wear increases with time and with the speed of rotation of the head. Fig. 2 shows the variation of the material lost by the dental mill due to wear depending on the rotation speed of the cutter head, for each of the four measurement times. It is observed that the wear increases with the rotation speed of the head and with time.

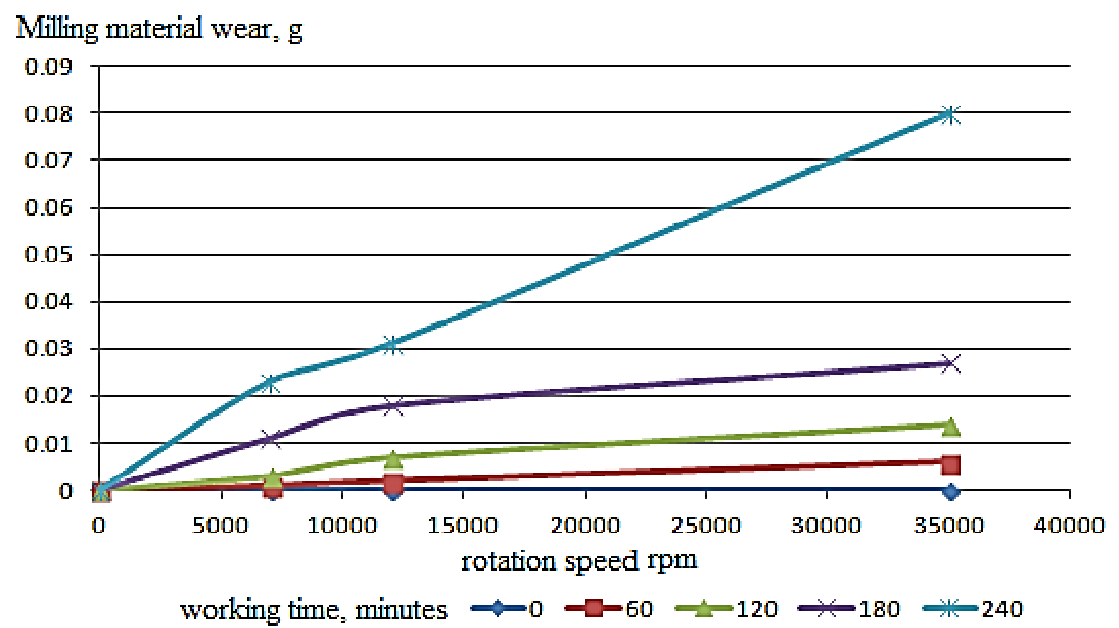

Fig. 2.Variation of the mass of wear of dental mill material, with the rotational speed of the mill, at different working times, including the initial state, $t=0$

The prolongation of the graphs between 0 and $7000 \mathrm{rpm}$ corresponds to the hypothesis that mechanical wear does not take place at zero speed.

Fig. 3 shows the variation of the angle of settlement, $\alpha$ alpha, with time, for three values of the rotation speed of the dental mill head. In general, it can be stated that this angle decreases over time and the decrease is even more intense at higher rotation speed of the dental cutter head.

In Fig. 4 the graphical representation is given of the variation of the clearance angle, $\gamma$, over time, for three values of the rotational speed of the tooth milling head. This angle increases over time and increases at faster rotational speed of the cutter head.

The variation of the sharpening angle in time for three values of the rotation speed of the dental mill head is represented graphically in Fig. 5. It is observed that the sharpening angle increases over time, at faster speed of rotation of the dental mill head.

The variation of the top circle area in time, for three values of the rotation speed of the dental mill head is represented graphically in Fig. 6. The increase in time is the faster at more intense rotational speed of the dental mill head. Experimental data have an appreciable deviation from this rule at average rotational speeds, which implies the need for additional experimental analysis. 


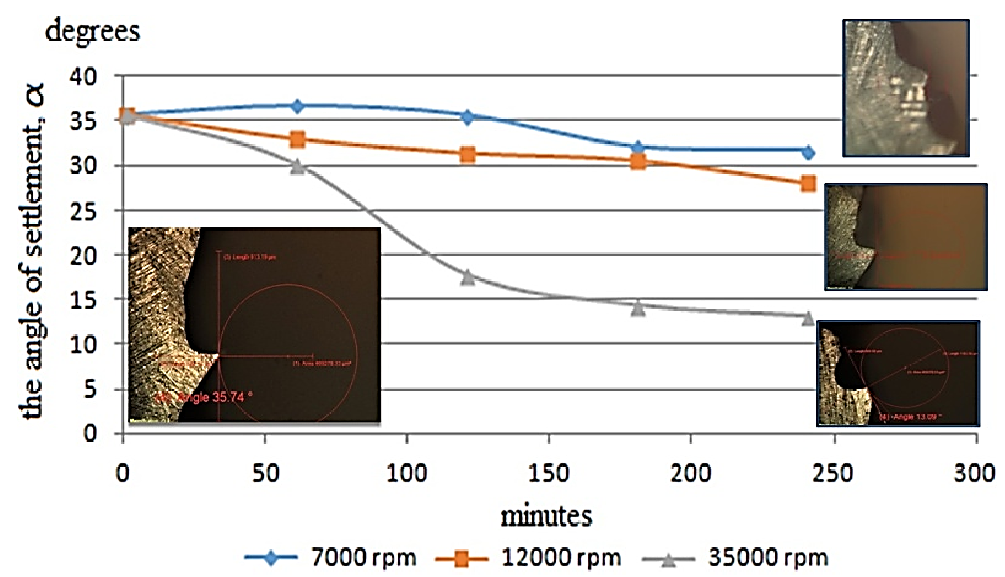

Fig. 3. Dependence of the value of the settlement angle (in degrees) depending on the operating time (in minutes) for three values of the rotational speed of the milling head (in rpm)

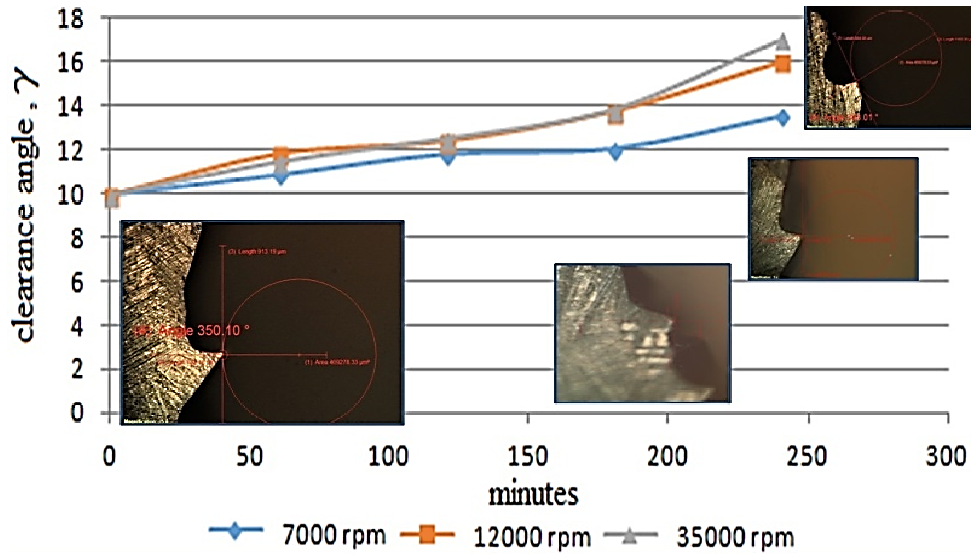

Fig. 4. Dependence of the clearance angle (in degrees) on the operating time (in minutes) for three values of the milling head rotation speed (in $\mathbf{r p m}$ )

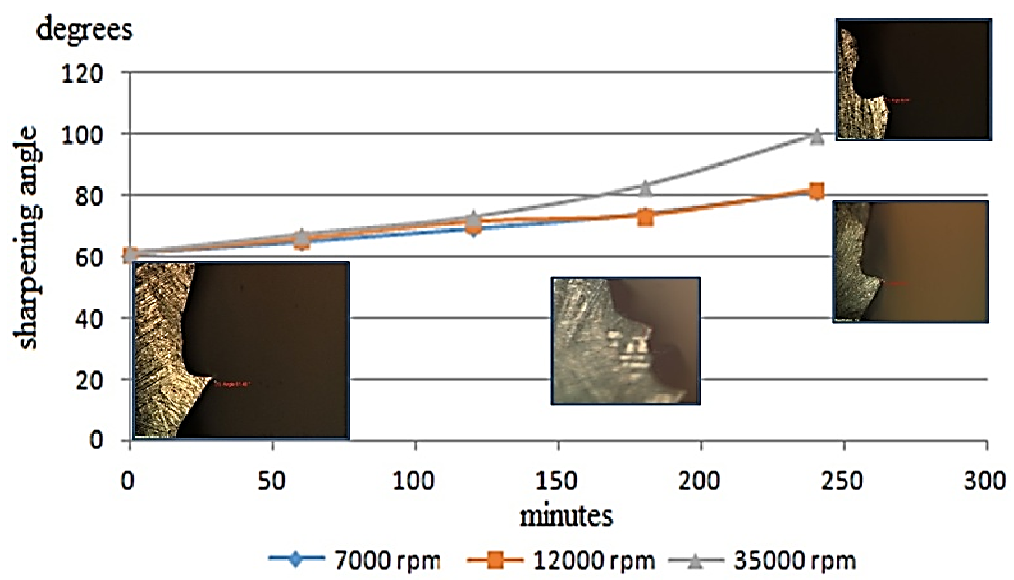

Fig. 5. Dependence of the sharpening angle (in degrees) on the operating time (in minutes) for three values of the milling head rotation speed (in rpm)

The variation of the top circle radius in time for three values of the rotation speed of the dental mill head is represented graphically in Fig. 7. The increase in time is faster at more intense rotational speed of the dental mill head. Experimental data have an appreciable deviation from this rule at average rotational speeds, which implies the need for additional experimental analysis. This phenomenon is similar to the behaviour of the top circle area. 


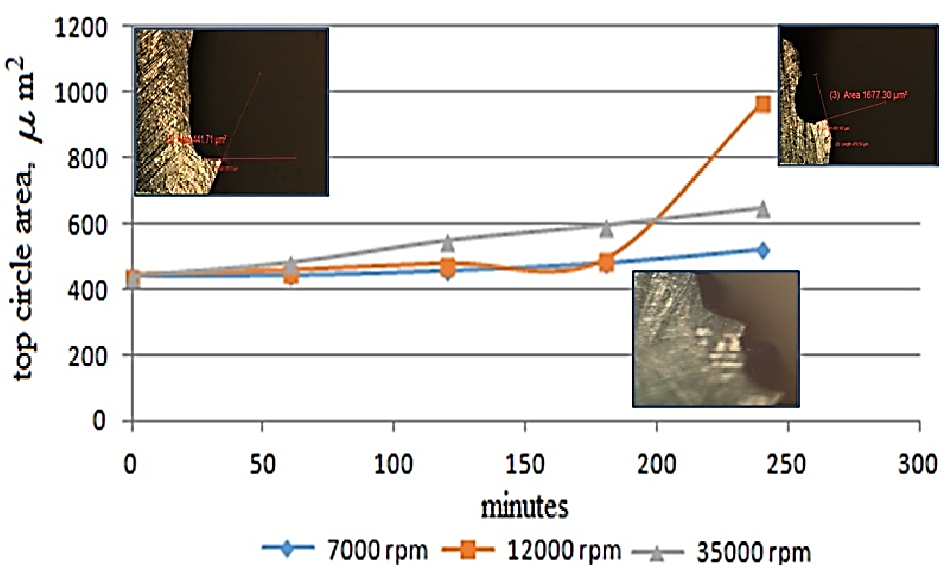

Fig. 6. Dependence of the top circle area angle (in degrees) on the operating time (in minutes) for three values of the milling head rotation speed (in $\mathbf{r p m}$ )

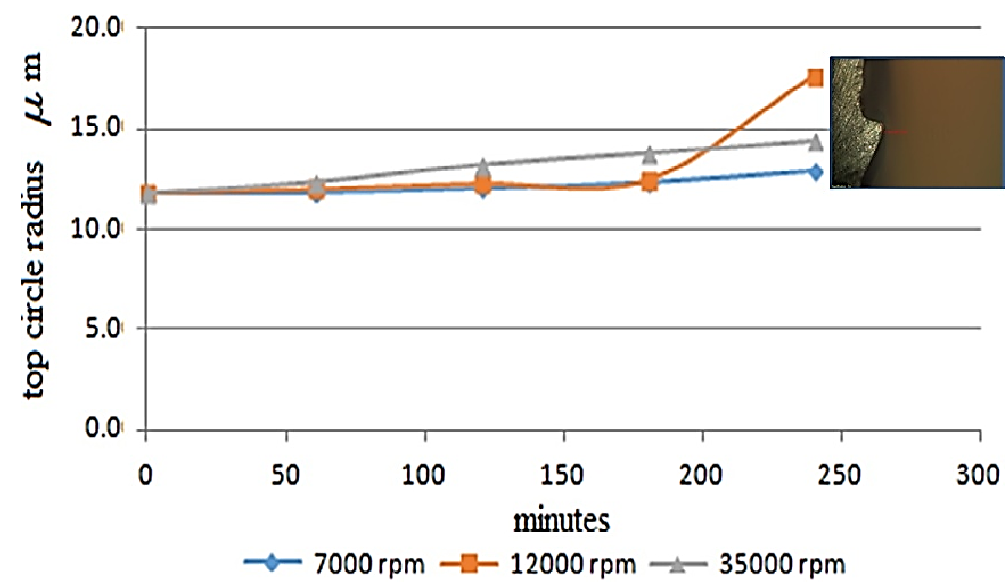

Fig. 7. Dependence of the top circle radius angle (in degrees) on the operating time (in minutes) for three values of the milling head rotation speed (in rpm)

As an alternative to the independent wear generation parameter, the product can be considered:

$$
\theta=t \cdot \omega .
$$

The parameter $\theta$ has the dimension of an angle, practically, the angular distance traveled in the rotation of the milling head. The angular velocity of formula (1) is related to the speed in Table 1 by the relation:

$$
\omega=\frac{\pi n}{30} .
$$

The relationships (1) and (2) and their interpretation reflect the similarity of the wear phenomenon of the head of the dental mill, with the phenomenon of wear of the vehicle tires, for example. Wear itself is a more complex phenomenon, a phenomenon in which two components participate equally, even though we call one active and one passive. For these reasons, the set of parameters that characterize the problem should be enriched with the parameters corresponding to the milled or passive material, respectively with structural characteristics of the mechanical strength of both components.

Given the above statement, among the most important parameters to be recorded in experiments is the mass of the milled material, i.e. the mass of material detached by milling from the body subjected to the milling modelling process. We note this mass with $m_{m}$. The value of this mass at the moment when the operator declares that the dental milling cutter becomes inefficient is very important in assessing the quality of the milling cutter, more precisely of the milling process.

A very important property of the materials that interact in the milling process is their hardness, both of the milling material and of the milled material. However, the variation of the hardness of the 
two materials in a complex experimentation program is a more distant objective in the study of the interaction between the milling cutter and the milled material, due to the material effort that exceeds us for the moment.

If we denote with $H_{f w}$ and $H_{m}$ the hardness of the milling material and the milled material, then we can try to establish some empirical relations based on the dimensional analysis between the parameters of the milling process.

One of the possible relations is of the form:

$$
\frac{m_{f w}}{m_{m}}=f\left(\alpha, \beta, \gamma, s, \omega, H_{f w}, H_{m}\right),
$$

where

$$
s=\frac{F}{p}
$$

is the contact surface between the milling cutter and the material being milled, $F$ is the pressing force of the milling cutter head on the contact material and $p$ is the contact pressure. It is found from the results of the experiments described above, that the three angles depend on $\omega$ and assuming in addition the dependence of the size of the contact surface on the same variable, the next relations are considered:

$$
\alpha=\alpha(\omega), \beta=\beta(\omega), \gamma=\gamma(\omega), s=s(\omega) .
$$

Relationships (5) can be described analytically by interpolation curves resulting using the statistical analysis of the experimental results, excepting the contact surface size $s$, for which additional measurements must be made or additional deductions are required. Using relations (5), formula (3) becomes:

$$
\frac{m_{f w}}{m_{m}}=f\left(\omega, H_{f w}, H_{m}\right) .
$$

If experimentally it is easier to control the pressing force of the dental mill head and the contact pressure, then (6) it can be written as:

$$
\frac{m_{f w}}{m_{m}}=f\left(\omega, F, p, H_{f w}, H_{m}\right),
$$

thus, in the calculation formula, the force of pressing the milling cutter on the milled material also appears as an important process control parameter.

An elementary variant of the relation (7), which can be tried for experimental data completed with information about the size of the contact surface or about the contact force and pressure, is the following:

$$
\frac{m_{f w}}{m_{m}}=f(\omega) \cdot \frac{F}{p} \cdot \frac{H_{f w}}{H_{m}},
$$

in which the empirical function $\mathrm{f}$ has the inverse dimension of the size of a surface, being determined from experimental data. In the statistical analysis we will try more complicated representations depending on several parameters, for example:

$$
\frac{m_{f w}}{m_{m}}=\left(f(\omega) \frac{F}{p}\right)^{\mu} \cdot\left(\frac{H_{f w}}{H_{m}}\right)^{v},
$$

where $\mu$ and $v$ are dimensionless parameters that will be established using statistical methods (classical least squares method or by numerical minimization of the attached functional).

An alternative way to find relationships between the parameters of the dental milling process can follow Archard's relationship [9], and the reasoning by which it was obtained. 


\section{Conclusions}

The experimental results obtained allow the extraction of some conclusions that aim at answers to the problems stated in the summary and introduction:

1. The results of the experimental tests show that the wear of the dental mills increases with the operating time (absolutely normal), with the working speed, with the pressing force. We expect this behaviour.

2. From the collected data, in addition, the growth rates of the wear (of the parameters that measure the wear) result. The direct determination from the experimental data the rates of increase of the wear is imprecise, affected by errors. For this reason, the values of the rates of increase of wear will be given only after the analysis and statistical modelling of the phenomenon.

3. The statistical analysis of the experimental data will allow, later, also fundamental physical considerations on a more comprehensive parametric space of the phenomenon of dental milling.

4. Taking into account presented formulas, it can be appreciated that a new experimental program will have to include the variations of the force of pressing of the milling head on the milled material, of the masses of the material lost by the dental milling and of the milled material. Also, although it is not simple, it is recommended to experiment for several values of the hardness of the materials involved in the process.

5. A special mentioning is required for the study of the contact surface, the pressing force and the contact pressure, linked by the relation described above. The measurement is complicated in this case, and the experimentation program increases even more by the variation of the parameter of the pressing force of the dental milling head on the milled material.

6. Finally we add a conclusion that refers to the thermo-mechanical properties of the materials in the milling interaction. As the rotational speeds of the milling head are high, local heating occurs in the milled area. Thus, temperature-dependent hardness changes may occur in the vicinity of the contact area. These variations must be considered in the context of a complex process of thermoelasto-plastic interaction, considering also the quality parameters of the processing of the milling material, because, under certain conditions, it can suffer irreversible damage.

\section{Acknowledgements}

This work has been funded by the European Social Fund from the Sectoral Operational Programme Human Capital 2014-2020, through the Financial Agreement with the title „Scholarships for entrepreneurial education among doctoral students and postdoctoral researchers (Be Entreprenor!)”, Contract No. 51680/09.07.2019 - SMIS code: 124539.

\section{References}

[1] Priarone P., C., Robiglio M., Settineri L., Tebaldo V. (2014). Milling and turning of titanium aluminides. Procedia CIRP 24 , 2014, pp. 62-67.

[2] Miyazaki T., Hotta Y., Kunii J., Kuriyama S., Tamaki Y., A review of dental CAD/CAM: Current status and future perspectives from 20 years of experience, Dental materials journal, 28, 2009, pp. 44-56.

[3] Riaz U., Liu C., Tan G., Li G., Yin N., Saeed M.J. Experimental Study on Cutting Force Comparison between Inner Cooling and Outer Cooling in Zig-zag Milling, IOP Publishing, IOP Conf. Series: Journal of Physics: Conf. Series 1187, 2019.

[4] Gouveia R.M., Silva F., J., G., Reis P., Baptista A., P., M. Machining Duplex Stainless Steel: Comparative Study Regarding End Mill Coated Tools. Coatings 6(4), 2016.

[5] Motorcu A.R., Kus A., Arslan R., Tekin Y., Ezentas R. Evaluation of tool life - tool wear in milling of inconel 718 superalloy and the investigation of effects of cutting parameters on surface roughness with taguchi method. Tehnicki vjesnik 20 (5), 2013, pp. 765-774.

[6] Nasri A, Slaimi J, Sai W.B,3D Parametric Modelling of Milling Cutter Geometry from Analytical Analysis. International Journal of Science, Technology and Society 4(2), 2016, pp. 35-40.

[7] Rehman G., U., I., Jaffery S., H., I., Khan M., Ali L., Khan A., Butt S.I. Analysis of Burr Formation in Low Speed Micro-milling of Titanium Alloy (Ti-6AI-4V). Mechanical Science, 2018, pp. 231-243. 
[8] Saracin A., Voicu Gh., Saracin I., Pandia O., Detemination of use of dental materials and stomatological frees on stand, conference "Engineering for Rural Development", Jelgava, 2019, pp. 1048-1052.

[9] Archard J., F., Hirst W., The Wear of Metals under Unlubricated Conditions, Proceedings of the Royal Society A-236 (1206), 1956, pp. 397-410. 